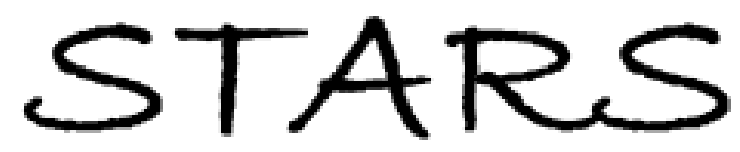

University of Central Florida

STARS

Faculty Bibliography 2000s

Faculty Bibliography

$1-1-2008$

\title{
Elastic modulus of viral nanotubes
}

Yue Zhao

University of Central Florida

Zhibin Ge

University of Central Florida

Jiyu Fang

University of Central Florida

Find similar works at: https://stars.library.ucf.edu/facultybib2000

University of Central Florida Libraries http://library.ucf.edu

This Article is brought to you for free and open access by the Faculty Bibliography at STARS. It has been accepted for inclusion in Faculty Bibliography 2000 s by an authorized administrator of STARS. For more information, please contactSTARS@ucf.edu.

\section{Recommended Citation}

Zhao, Yue; Ge, Zhibin; and Fang, Jiyu, "Elastic modulus of viral nanotubes" (2008). Faculty Bibliography 2000s. 1213.

https://stars.library.ucf.edu/facultybib2000/1213

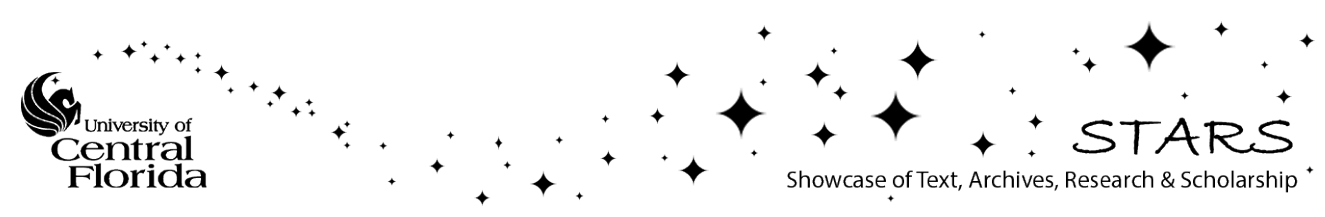




\title{
Elastic modulus of viral nanotubes
}

\author{
Yue Zhao, ${ }^{1}$ Zhibin Ge, ${ }^{2}$ and Jiyu Fang ${ }^{1, *}$ \\ ${ }^{1}$ Advanced Materials Processing and Analysis Center and Department of Mechanical, Materials, and Aerospace Engineering, \\ University of Central Florida, Orlando, FL 32816, USA \\ ${ }^{2}$ College of Optics and Photonics, University of Central Florida, Orlando, Florida 32816, USA \\ (Received 24 March 2008; revised manuscript received 3 June 2008; published 17 September 2008)
}

\begin{abstract}
We report an experimental and theoretical study of the radial elasticity of tobacco mosaic virus (TMV) nanotubes. An atomic force microscope tip is used to apply small radial indentations to deform TMV nanotubes. The initial elastic response of TMV nanotubes can be described by finite-element analysis in $5 \mathrm{~nm}$ indentation depths and Hertz theory in $1.5 \mathrm{~nm}$ indentation depths. The derived radial Young's modulus of TMV nanotubes is $0.92 \pm 0.15 \mathrm{GPa}$ from finite-element analysis and 1.0 $\pm 0.2 \mathrm{GPa}$ from the Hertz model, which are comparable with the reported axial Young's modulus of 1.1 GPa [Falvo et al., Biophys. J. 72, 1396 (1997)].

DOI: 10.1103/PhysRevE.78.031914

PACS number(s): 87.15.La, 87.15.A-, 87.64.Dz
\end{abstract}

\section{INTRODUCTION}

Tobacco mosaic virus (TMV) is a hollow tubular structure formed by the self-assembly of 2130 identical protein subunits (molecular weight $17.5 \mathrm{kDa}$ ) in a right-handed helical motif around a single-strand genomic RNA which forms a hairpin loop structure [1]. It has a length of $\sim 300 \mathrm{~nm}$ and an outer diameter of $18 \mathrm{~nm}$. The diameter of its cavity is $4 \mathrm{~nm}$. The structure of TMV nanotubes has been characterized at atomic resolution by x-ray fiber diffraction [2] and cryoelectron microscopy $[3,4]$. Recently, there has been great interest in using TMV nanotubes with charged amino-acid surfaces as a template for synthesizing bioinorganic nanostructures for electronic [5-12] and optical [13,14] applications. For the templated-direction metallization reactions, the rigidity of TMV nanotubes determines whether they can be used as a template. The use of TMV nanotubes as a template in some cases requires them to be repositioned and aligned into a desired conformation [15-17]. Therefore, an understanding of the radial elasticity of TMV nanotubes is important in developing their applications in the templated synthesis of bioinorganic nanostructures.

Recently, the atomic force microscope (AFM) is emerging as a powerful tool in studying nanomechanics of viral nanoparticles because it combines high sensitivity in applying and measuring forces and high precision in positioning a tip relative to viral nanoparticles with the ability to operate in physiological environments [18-23]. The measurement of elastic response to the indentation of viral nanoparticles allows us to obtain quantitative values of their elastic modulus. Although the bending rigidity of TMV nanotubes has been previously studied, there is controversy surrounding the reported values of the axial Young's modulus. For example, Falvo et al. [24] bent individual TMV nanotubes absorbed on graphite substrates with an AFM tip through a nanomanipulator system under ambient conditions. By using a mechanical model that takes into account the lateral force applied by the scanning AFM tip and the frictional force by the graphite substrate, they estimated the axial Young's modulus of TMV nanotubes

\footnotetext{
*jfang@mail.ucf.edu
}

to be $\sim 1.1 \mathrm{GPa}$. Schmatulla et al. [25] reportedly used an AFM tip to apply a point force to bend a TMV nanotube that was assumed to be suspended from a mica substrate at one end by crossing another TMV nanotube. The axial Young's modulus of TMV nanotubes estimated by this bending method is $\sim 5 \mathrm{GPa}$. The large difference in the reported values of the axial Young's modulus might be a result of the wrong assumptions made in these bending measurements. Furthermore, the elastic properties of TMV nanotubes should be associated with not only the properties of individual protein subunits, but also the interactions between them. For a helical arrangement of protein subunits, the flexural rigidity of TMV nanotubes is expected to be dominated by the protein subunit interactions between successive turns, whereas radial indentation experiments should be sensitive to the interactions of protein subunits along the helical turns. The measurements of axial and radial Young's moduli of TMV nanotubes might shine light on the interactions of protein subunits. A detailed understanding of the radial mechanical properties of TMV nanotubes is also critical for evaluating their suitability for the templated-direction metallization reactions. Here, we study the radial elasticity of TMV nanotubes by local AFM indentations coupled with finite-element analysis and Hertz theory. The radial Young's moduli of the TMV nanotubes derived from both models are comparable, and also in good agreement with the axial Young's modulus reported by Falvo et al. [25].

\section{EXPERIMENTAL METHODS}

Tobacco mosaic virus (common strain, ATCC catalog no. PV-135P) was dissolved in $10 \mathrm{~m} M$ sodium phosphate buffer at $p \mathrm{H}$ 7.0. A drop $(100 \mu \mathrm{l})$ of the TMV solution was placed onto freshly cleaved mica and then left to absorb on the substrate for $30 \mathrm{~min}$ in air at room temperature. Finally, a drop $(100 \mu \mathrm{l})$ of buffer was added on the sample to ensure complete immersion of the cantilever in an opened liquid cell. An atomic force microscope (Dimension 3100, Digital Instruments) with an opened loop scanner and software version 6.12r1 was used to image TMV nanotubes adsorbed on mica and study their mechanical properties in contact mode. Silicon nitride cantilever tips (Nanosensors) with a curvature 

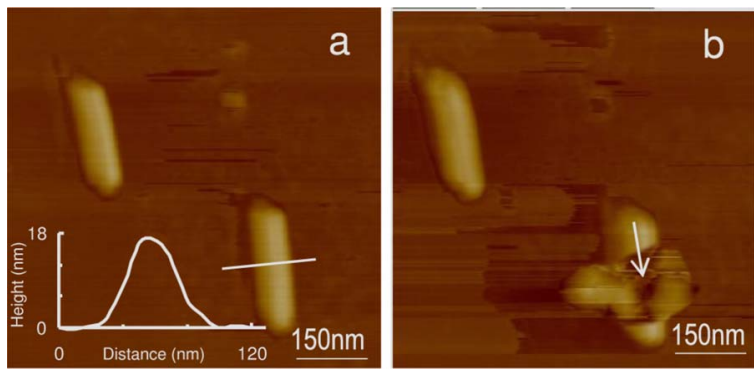

FIG. 1. (Color online) AFM images of TMV nanotubes (a) before and (b) after indentation. The high profile across a nanotube is inset in (a). The white arrow shown in (b) indicates the collapse of the nanotube after indentation.

radius of about $15 \mathrm{~nm}$ according to the manufacturer were employed. The spring constants of silicon nitride cantilevers were determined by measuring their thermal fluctuations [26] to be $\sim 0.051 \mathrm{~N} / \mathrm{m}$. AFM images were obtained in contact mode at a scanning rate of $0.5 \mathrm{~Hz}$ and a $512 \times 512$ pixel size. To avoid the tip slipping off the top surface of the TMV nanotube during indentation, low scan rates from 0.297 to $0.197 \mathrm{~Hz}$, which correspond to forward velocities from 297 to $197 \mathrm{~nm} / \mathrm{s}$, were used. Force- $Z$ piezo distance (FZ) curves were recorded as the AFM cantilever approached the TMV nanotubes.

During the finite-element simulation, the TMV nanotubes were modeled as hollow tubes made of homogeneous materials, sitting on a flat rigid surface and loaded by a rigid spherical indenter with radius of $15 \mathrm{~nm}$, similar to the size of the AFM tips used in our indentation experiments. The thickness:radius:length ratio $(t: r: l)$ of the modeled TMV nanotube is 7:9:48. Considering the axial symmetry of the indentation contact, the model was reduced to one quarter by using the two mirror symmetry planes intersecting at the loading point, one parallel and one perpendicular to the tube axis. This quarter was meshed into 3000-4500 three-dimensional brick elements and subjected to the symmetrical boundary conditions. The postprocessing was done with the software ABAQUS 6.6. Loading was simulated by prescribing a downward movement of the rigid spherical indenter, which is calculated in 100 increments. The AFM tip-TMV tube and the TMV tube-rigid substrate contacts are modeled as frictionless.

\section{RESULTS AND DISCUSSION}

Figure 1(a) shows an AFM image of TMV nanotubes adsorbed on mica. The image was taken in contact mode in $10 \mathrm{~m} M$ sodium phosphate buffer solution at $p \mathrm{H} \mathrm{7.0.} \mathrm{The}$ force employed for the scan was $\sim 1.4 \mathrm{nN}$. The length of the TMV nanotubes is $\sim 250 \mathrm{~nm}$. Their apparent widths in the AFM image are broadened by the finite size of the AFM tips. The high profile shows that the TMV nanotube has a cylindrical shape with a height of $17.2 \mathrm{~nm}$ (see inset), very close to the diameter $(18 \mathrm{~nm})$ of TMV nanotubes measured by transmission electron microscopy [3], suggesting that the TMV tube is not compressed at the force employed for scanning or grossly distorted by the adsorption during the sample

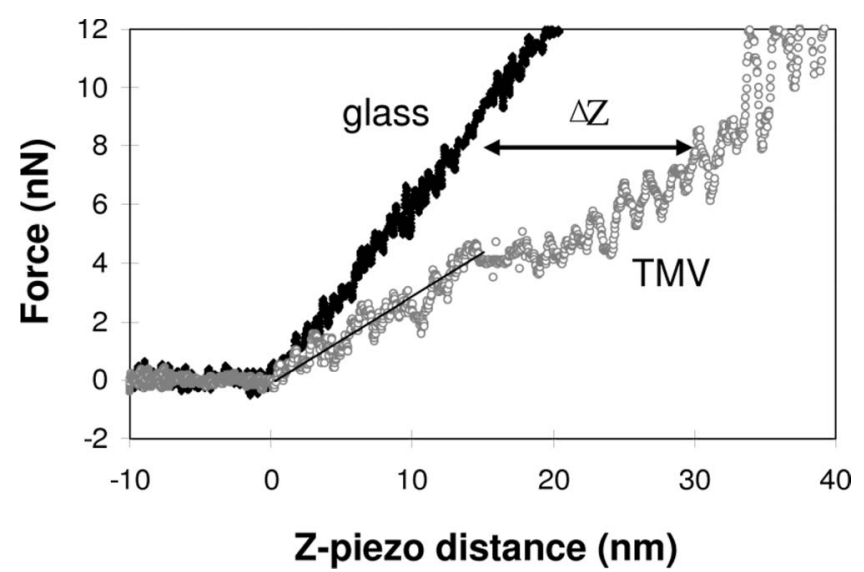

FIG. 2. Force $-Z$ piezo distance (FZ) curve (gray) taken on a TMV nanotube, together with the FZ curve (black) on a glass substrate. Both FZ curves were performed under AFM contact mode in a liquid cell with the same AFM tip.

preparation. The distortion or removal of TMV nanotubes from mica by the AFM tip was observed when the force employed for scanning was above $4 \mathrm{nN}$. In our experiments, all AFM images of TMV tubes were taken at a force below $4 \mathrm{nN}$. Due to the thermal drift, exact control of the indentation position is difficult. To precisely position an AFM tip on the top of a TMV nanotube, we first located it by lowering the scanning speed to $0.3 \mu \mathrm{m} / \mathrm{s}$ and gradually reducing the scan size to $5 \times 5 \mathrm{~nm}^{2}$. Since the AFM cantilever is at an angle relative to the sample surface, a lateral tip motion during indentation measurements can take place. The $X$-rotate parameter of software version $6.12 \mathrm{r} 1$ is designed to prevent the cantilever from plowing the surface along the $X$ direction by compensating the lateral tip motion, while it indents the sample surface in the $Z$ direction. In our experiments, the $X$-rotate parameter was set to be $22^{\circ}$ to ensure vertical indentations, and therefore further prevent the lateral friction force. After the indentation, the TMV nanotubes were reimaged by AFM to ensure that the indentation was preformed on the tube. As can be seen in Fig. 1(b), the indented TMV nanotube is collapsed.

Figure 2 shows a typical force- $Z$ piezo distance curve taken on a collapsing TMV nanotube at a velocity of $197 \mathrm{~nm} / \mathrm{s}$ with a maximum force of $12 \mathrm{nN}$, together with the corresponding FZ curve on a mica substrate taken with the same cantilever tip. Both FZ curves were shifted along the $Z$ piezo distance axis to set the tip-sample contact at the same point. The mica substrate can be considered as an infinitely stiff material, compared to the AFM cantilever. So the linear FZ curve taken on the mica substrate can be used as a reference. The horizontal distance $(\Delta Z)$ between the forward FZ curves at a given force represents the actual indentation depth of the TMV tube by the AFM tip. As can be seen from Fig. 2, the forward FZ curve on the TMV nanotube is linear up to a force of $\sim 4.4 \mathrm{nN}$, corresponding to an actual indentation depth of $\sim 5 \mathrm{~nm}$, i.e., $\sim 27 \%$ of the original TMV diameter. At this point there is a sudden change in the slope, suggesting the collapse of the TMV nanotube under the indentation. The collapsed TMV nanotube shows a nonlinear response as the force increases. Above $8 \mathrm{nN}$, the slope of the 
FZ curve collected on the collapsed TMV nanotube reaches that on the mica substrate, meaning that the substrate effect became dominant. After collapsing, the FZ curve on the TMV nanotube cannot be retraced when the tip is withdrawn from its surface. In subsequent indentations the initial linear response in the FZ curve disappeared. However, if multiple indentations are carried out within the linear response regime of a TMV nanotube, the FZ curve is reversible with a very small hysteresis.

An effective spring constant of $0.34 \pm 0.03 \mathrm{~N} / \mathrm{m}$ was measured from the slopes of the FZ curves in their linear elastic regime, which were collected on 22 collapsing TMV nanotubes with five AFM tips from the same manufacturer. The relatively large standard deviation likely involves the deviation of the indentation positions away from the centers of the TMV nanotubes, the variation of the spring constants of the cantilevers, and the shape changes of TMV nanotubes. The effective sprint constant $\left(k_{\text {eff }}\right)$ comprises the spring constants of the TMV nanotubes $\left(K_{\mathrm{TMV}}\right)$ and the cantilevers $\left(K_{\mathrm{can}}\right)$. If the indentation system is modeled as two springs arranged in a series, the point spring constant (stiffness) of TMV nanotubes can be calculated according to Hooke's law:

$$
k_{\mathrm{TMV}}=\frac{k_{\mathrm{can}} k_{\mathrm{eff}}}{k_{\mathrm{can}}-k_{\mathrm{eff}}} .
$$

Using Eq. (1), we calculate that the point spring constant (stiffness) of TMV nanotubes is $0.80 \pm 0.13 \mathrm{~N} / \mathrm{m}$.

The wall thickness:radius ratio $(t: r)$ of TMV nanotubes is 0.78 , which is far away from the thin-shell range of 0.002 . Therefore, we used finite-element analysis to calculate the Young's modulus of the TMV nanotubes. During the simulation, TMV nanotubes were modeled as a thick-walled cylinder with an outer radius of $9 \mathrm{~nm}$ and an inner radius of $2 \mathrm{~nm}$ sitting on a flat rigid surface and loaded by a spherical rigid indenter with a radius of $15 \mathrm{~nm}$ similar to the diameter of the AFM tip used in our experiments. The finite-element model allows us to follow the deformation of TMV nanotubes as they are indented and determine the local stress in the tube walls. Figure 3 shows a sequence of deformed shapes of a modeled TMV nanotube, color coded by von Mises stress at several intervals during the indentation process. The deformation zone on the tube surface is approximately the same as the contact area of the indenter. At the beginning of the indentation the von Mises stress is built up around the contact point in the upper region of the TMV nanotube [Fig. 3(a)] and then spreads along the radial direction [Fig. 3(b)]. The magnitude of the stress decreases through the tube wall away from the contact region. The stress then starts to develop in the lower region of the nanotube which is in contact with the mica substrate [Fig. 3(c)]. As the indentation continues, the tube wall of the upper region is severely deformed and the hollow core of the nanotube is squeezed [Fig. 3(d)]. At $5 \mathrm{~nm}$ indentation, the upper region of the tube wall buckles away from the AFM tip, leading to the opening of a small gap between the AFM tip and the tube [Fig. 3(e)]. Because of the buckling of the tube, the maximum stress appears at the inner surface. During the simulation process, we varied the Young's modulus until the simulated force-indentation (FI) curve is best fitted with the (a)
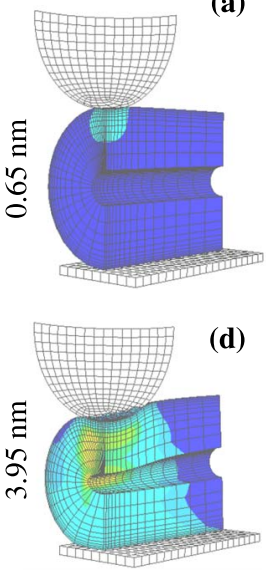

(b)

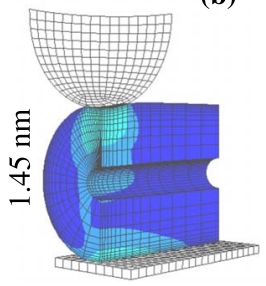

(e)

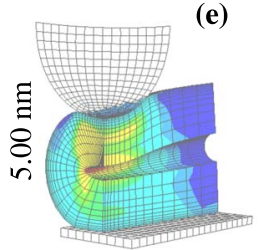

(c)
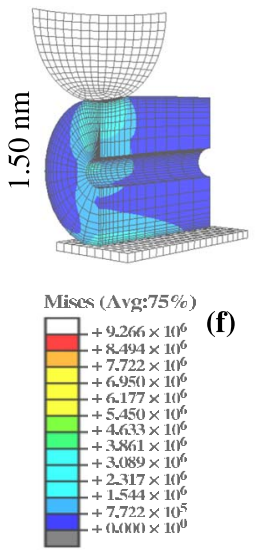

FIG. 3. (Color online) Simulated deformation of a nanotube with an outer radius of $9 \mathrm{~nm}$ and an inner radius of $2 \mathrm{~nm}$ at indentations of (a) 0.65 , (b) 1.45 , (c) 1.50 , (d) 3.95 , and (e) $5.00 \mathrm{~nm}$, respectively. The von Mises stress is indicated by color contours (f).

experimental data collected from 22 collapsing TMV nanotubes (Fig. 4). The best fitting gives a Young's modulus of $0.92 \pm 0.15 \mathrm{GPa}$.

In addition, we note that the FI curve derived from the finite-element model is nonlinear at small indentation depths $(<1.5 \mathrm{~nm})$ (Fig. 4). Beyond this initial small indentation region, the simulated FI curve becomes linear. As can be seen from Figs. 3(a) and 3(b), at the small initial indentations the von Mises stress is concentrated in the upper region of the tube wall and decays through the tube wall away from the contact region. It is likely that the local compression of the tube wall, rather than the deformation of the TMV hollow core, is dominant at the initial small indentations. Thus a nonlinear dependence of the force on the indentation depth is expected. When the force is above $1.5 \mathrm{nN}$, a deformation of the hollow core is involved, resulting in the linear dependence of the force on the indentation depth. We applied the Hertz model to the initial nonlinear deformation by considering the contact between the AFM tip and the TMV nanotube as a contact between a rigid sphere and an isotropic cylinder. The relationship between the spring constant of TMV nanotubes and their Young's modulus can be described as follows [27]:

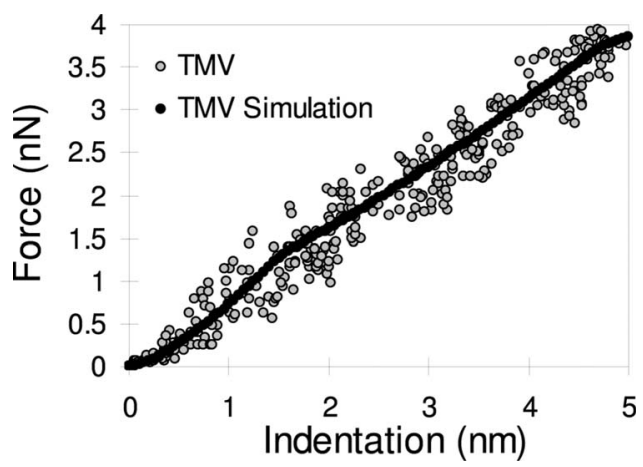

FIG. 4. Experimental data (circular dots) of force vs indentation for TMV nanotubes within $5 \mathrm{~nm}$ indentation. Young's modulus was calculated from the best fitting of simulated force-indentation data (square dots) with finite-element analysis. 


$$
k_{\text {tube }}=\beta\left(\frac{R F}{\frac{3}{4}\left(\frac{1-v_{\text {tip }}^{2}}{E_{\text {tip }}}+\frac{1-v_{\text {tube }}^{2}}{E_{\text {tube }}}\right)}\right)^{1 / 3},
$$

where $1 / R=1 / R_{\text {tip }}+\left(1 / 2 R_{\text {tube }}\right) \nu_{\text {tip,tube }}$ and $E_{\text {tip,tube }}$ are the Poisson ratio and radial Young's modulus of the AFM tip and the TMV nanotube. $\beta$ is a coefficient that takes the geometrical aspect of the contact area into account and can be expressed as $\beta=(\pi / 2 k)^{2 / 3} E\left(\sqrt{1-k^{2}}\right)^{1 / 3} / K\left(\sqrt{1-k^{2}}\right)$, where $k$ is a parameter taking into account the elliptic shape of the contact determined by solving the expression: $1+R_{\text {tip }} / R_{\text {tube }}$ $=\left(1 / k^{2}\right)\left[E\left(\sqrt{1-k^{2}}\right)-K\left(\sqrt{1-k^{2}}\right)\right] /\left[K\left(\sqrt{1-k^{2}}\right)-E\left(\sqrt{1-k^{2}}\right)\right]$,

where $E(k)$ and $K(k)$ are the complete intergral of elliptic $E$ and elliptic $K$ defined by $E(k)=\int_{0}^{\pi / 2} \sqrt{1-k^{2} \sin ^{2} \theta} d \theta$ and $K(k)=\int_{0}^{\pi / 2} d \theta / \sqrt{1-k^{2} \sin ^{2} \theta}$.

The relation of the loading force $(F)$ and the corresponding indentation depth $(\Delta Z)$ is given by Hooke's law:

$$
F=K_{\text {tube }} \Delta Z \text {. }
$$

By substituting Eq. (3) into Eq. (2), we get

$$
F=\left(\frac{R}{\frac{3}{4}\left(\frac{1-v_{\text {tip }}^{2}}{E_{\text {tip }}}+\frac{1-v_{\text {tube }}^{2}}{E_{\text {tube }}}\right)}\right)^{1 / 2}(\beta \Delta Z)^{3 / 2} .
$$

The Poisson ratio and Young's modulus of the silicon nitride tip are $\nu_{\text {tip }}=0.27$ and $E_{\text {tip }}=155 \mathrm{GPa}$ [28]. The Poisson ratio of the TMV nanotube is taken as $\nu_{\text {tube }}=0.48$, a mean value of common biomaterials. Thus, in Eq. (4), the Young's modulus of TMV nanotubes $\left(E_{\text {tube }}\right)$ is the only fitting parameter. Figure 5 shows the fitting curves for the first $1.5 \mathrm{~nm}$ indentation data. The fitting gives a radial Young's modulus of TMV nanotubes of $1.0 \pm 0.2 \mathrm{GPa}$, which is comparable with that calculated from finite-element analysis.

The radial Young's modulus of TMV nanotubes calculated from both models is very close to the axial Young's modulus measured by the bending experiment [24]. The intersubunit electrostatic interactions between successive helical turns and along the helical turns are expected to dominate the axial bending and the radial deformation of TMV nanotubes, respectively. The consistency between the measured radial and axial Young's moduli may suggest that the interactions of protein subunits are isotropic in TMV nanotubes. Our results may also suggest that the RNA with a hairpin loop located at a radius of $\sim 6 \mathrm{~nm}$ does not produce a profound impact in the Young's modulus of TMV nanotubes.

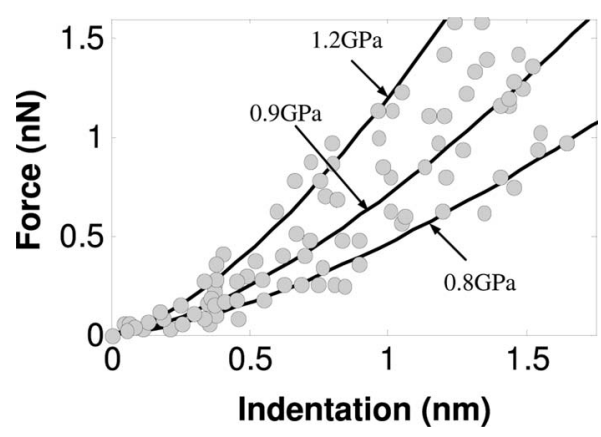

FIG. 5. Experimental data (circular dots) of force vs indentation for TMV nanotubes in $1.5 \mathrm{~nm}$ indentation. The solid lines represent the fits of the experimental data with the Hertz model. The Young's modulus from these optimized fitting results is $1.2,0.9$, and $0.8 \mathrm{GPa}$, respectively.

Compared to other biological tubule structures, we find that the Young's modulus of TMV nanotubes is close to that of lipid tubules $(0.7-1.0 \mathrm{GPa})[22,29]$ and protein microtubules $(0.8 \mathrm{GPa})$ [30]. But they are stiffer than $\alpha$-lactalbumine protein tubes, which have a Young's modulus of $0.1 \mathrm{GPa}$ [31], and softer than diphenylalanine peptide nanotubes with a Young's modulus of $19 \mathrm{GPa}$ [32]. Compared to other virus particles with spherical shapes, the Young's modulus of TMV nanotubes is close to that of plant cowpea chlorotic mottle $(0.9 \mathrm{GPa})$ [19], but smaller than that of bacteriophage $\phi 29(1.8 \mathrm{GPa})[18]$.

\section{CONCLUSION}

The radial elastic properties of TMV nanotubes have been studied by applying small indentations onto them with AFM tips. We find that the initial elastic response of TMV nanotubes to small radial indentations can be described by finiteelement analysis in $5 \mathrm{~nm}$ indentation depth and Hertz theory in $1.5 \mathrm{~nm}$ indentation depth. The derived radial Young's modulus of TMV nanotubes is $0.92 \pm 0.15 \mathrm{GPa}$ from finiteelement analysis and 1.0 $\pm 0.2 \mathrm{GPa}$ from the Hertz model, respectively.

\section{ACKNOWLEDGMENT}

This work was supported by the National Science Foundation Grant No. (CMMI 0726478).
[1] A. Klug, Philos. Trans. R. Soc. London, Ser. B 354, 531 (1999).

[2] K. Namba, R. Pattanayek, and G. Stubbs, J. Mol. Biol. 208, 307 (1989).

[3] T. W. Jeng, R. A. Crowther, G. Stubbs, and W. Chiu, J. Mol. Biol. 205, 251 (1989).

[4] D. R. Diaz-Avalos and L. D. Caspar, J. Mol. Biol. 197, 67 (2000).
[5] W. Shenton, T. Douglas, M. Young, G. Stubbs, and S. Mann, Adv. Mater. (Weinheim, Ger.) 11, 253 (1999).

[6] E. Dujardin, C. Peet, G. Stubbs, J. N. Culver, and S. Mann, Nano Lett. 3, 413 (2003).

[7] M. Knez, M. Sumser, A. M. Bittner, C. Wege, H. Jeske, T. P. Martin, and K. Kern, Adv. Funct. Mater. 14, 116 (2004).

[8] V. A. Fonoberov and A. A. Balandin, Nano Lett. 5, 1920 (2005). 
[9] W. L. Liu, K. Alim, A. A. Balandin, D. M. Mathews, and J. A. Dodds, Appl. Phys. Lett. 86, 253108 (2005).

[10] M. Knez, A. Kadri, C. Wege, U. Glosele, H. Jeske, and K. Nielsch, Nano Lett. 6, 1172 (2006).

[11] M. Endo, H. Wang, M. Fujitsuka, and T. Majima, Chem.-Eur. J. 12, 3735 (2006).

[12] R. Tsukamoto, M. Muraoka, M. Seki, H. Tabata, and I. Yamashita, Chem. Mater. 19, 2389 (2007).

[13] T. L. Schlick, Z. Ding, E. W. Kovacs, and M. B. Francis, J. Am. Chem. Soc. 127, 3718 (2005).

[14] R. A. Miller, A. D. Presley, and M. B. Francis, J. Am. Chem. Soc. 129, 3104 (2007).

[15] R. J. Tseng, C. Tsai, L. Ma, J. Ouyang, C. S. Ozkan, and Y. Yang, Nat. Nanotechnol. 1, 72 (2006).

[16] D. M. Kuncicky, R. R. Naik, and O. D. Velev, Small 2, 1462 (2006).

[17] H. Yi, S. Nisar, S. Y. Lee, M. A. Powers, W. E. Bentley, G. F. Payne, R. Ghodssi, G. W. Rubloff, M. T. Harris, and J. N. Culver, Nano Lett. 5, 1931 (2005).

[18] I. L. Ivanovska, P. J. de Pablo, B. Ibarra, G. Sgalari, F. C. Mackintosh, J. L. Carrascoa, C. F. Schmidt, and G. J. L. Wuite, Proc. Natl. Acad. Sci. U.S.A. 101, 7600 (2004).

[19] J. P. Michel, I. L. Ivanovska, M. M. Gibbons, W. S. Klug, C. M. Knobler, G. J. L. Wuite, and C. F. Schmidt, Proc. Natl. Acad. Sci. U.S.A. 103, 6184 (2006).

[20] W. S. Klug, R. F. Bruinsma, J. P. Michel, C. M. Knobler, I. L. Ivanovska, C. F. Schmidt, and G. J. L. Wuite, Phys. Rev. Lett. 97, 228101 (2006).

[21] C. Carrasco, A. Carreira, I. A. T. Schaap, P. A. Serena, J.
Gómez-Herrero, M. G. Mateu, and P. J. de Pablo, Proc. Natl. Acad. Sci. U.S.A. 103, 13706 (2006).

[22] Y. Zhao, K. Tamhane, X. Zhang, L. An, and J. Y. Fang, ACS Nano. 2, 1466 (2008)

[23] N. Kol, M. Gladnikoff, D. Barlam, R. Z. Shneck, A. Rein, and I. Rousso, Biophys. J. 91, 767 (2006).

[24] M. R. Falvo, S. Washburn, R. Superfine, M. Finch, F. P. Brooks, Jr., V. Chi, and R. M. Taylor II, Biophys. J. 72, 1396 (1997).

[25] A. Schmatulla, N. Maghelli, and O. Marti, J. Microsc. 225, 264 (2007).

[26] J. L. Hutter and J. Bechhoefer, Rev. Sci. Instrum. 64, 1868 (1993)

[27] A. P. Boresi and O. M. Sidebottom, Advanced Mechanics of Materials (Wiley, New York, 1993).

[28] R. W. Carpick, M. Enachescu, D. F. Ogletree, and M. Salmeron, in Fracture and Deuctive vs. Brittle Behavior-Theory, Modeling and Experiment, edited by G. E. Beltz, R. L. Lumberg Selinger, M. P. Marder, and K.-S. Kim, MRS Symposia Proceedings No. 539 (Materials Research Center, Pittsburgh, 1999).

[29] Y. Zhao, A. An, and J. Y. Fang, Nano Lett. 7, 1360 (2007).

[30] P. J. de Pablo, I. A. T. Schaap, F. C. MacKintosh, and C. F. Schmidt, Phys. Rev. Lett. 91, 098101 (2003).

[31] J. F. Graveland-Bikker, I. A. T. Schaap, C. F. Schmidt, and C. G. de Kruif, Nano Lett. 5, 616 (2006).

[32] N. Kol, L. Adler-Abramovich, D. Barlam, R. Z. Shneck, E. Gazit, and I. Rousso, Nano Lett. 4, 1343 (2005). 